\title{
OUT-SPHERE DECODER FOR NON-COHERENT ML SIMO DETECTION AND ITS EXPECTED COMPLEXITY
}

\author{
M. Stojnic and B. Hassibi \\ California Institute of Technology, Pasadena, CA
}

\begin{abstract}
In multi-antenna communication systems, channel information is often not known at the receiver. To fully exploit the bandwidth resources of the system and ensure the practical feasibility of the receiver, the channel parameters are often estimated and then employed in the design of signal detection algorithms. However, sometimes communication can occur in an environment where learning the channel coefficients becomes infeasible. In this paper we consider the problem of maximum-likelihood (ML)-detection in singleinput multiple-output (SIMO) systems when the channel information is completely unavailable at the receiver and when the employed signalling at the transmitter is $q$-PSK. It is well known that finding the solution to this optimization requires solving an integer maximization of a quadratic form and is, in general, an NP hard problem. To solve it, we propose an exact algorithm based on the combination of branch and bound tree search and semi-definite program (SDP) relaxation. The algorithm resembles the standard sphere decoder except that, since we are maximizing we need to construct an upper bound at each level of the tree search. We derive an analytical upper bound on the expected complexity of the proposed algorithm.
\end{abstract}

\section{INTRODUCTION}

Multi-antenna wireless communication systems are capable of providing reliable data transmission at very high rates. The channel in such systems is, in principle, unknown to the receiver and needs to be estimated either prior to or concurrently with the detection of the transmitted signal. However learning channel coefficients requires time which in environments with rapidly changing conditions can be impractical. In this paper we study the problem of ML detection when the channel information is unavailable at the receiver. The system, that we study has a single transmit antenna and multiple receive antennas.

This work was supported in part by the National Science Foundation under grant no. CCR-0133818, by Caltech's Lee Center for Advanced Networking, and by a grant from the David and Lucille Packard Foundation.
We assume a standard flat-fading channel model for multiantenna systems (see Figure 1),

$$
X=\sqrt{\rho m} \mathbf{s h}+W
$$

where $m$ denotes the number of receive antennas. The number of the transmitted antennas is 1. $\rho$ is the signal-to-noise ratio (SNR), $X$ is a $m \times m$ matrix of the received symbols, $\mathrm{s}$ is a $m \times 1$ transmitted symbol vector comprised of components $s_{i}$ for which it holds that $s_{i}=\frac{1}{\sqrt{T}} e^{\frac{j 2 r \pi}{q}}, r=1, \ldots, q$, $q$ is an integer power of $2, \mathbf{h}$ is an $1 \times m$ channel matrix whose components are independent, identically distributed (i.i.d.) zero-mean, unit-variance complex/real Gaussian random variables and that is constant for $m$ channel uses, and $W$ is an $m \times m$ noise matrix whose components are i.i.d. zero-mean, unit-variance complex/real Gaussian random variables. Furthermore, we assume that the components of $\mathbf{h}$ and $W$ are uncorrelated, which is often the case in practice.[We will assume that $\mathbf{h}$ and $W$ are complex if $q \geq 4$ and real if $q=2$.]

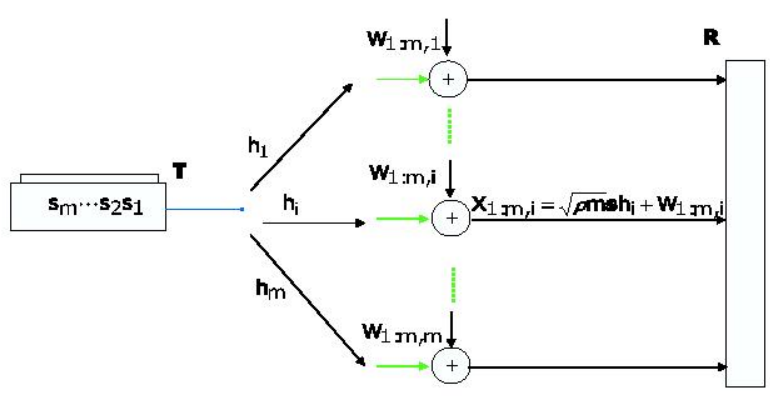

Fig. 1. SIMO system model

In the next section, we recall what the criterion for noncoherent ML-signal detection is and propose an efficient algorithm for finding its approximate solution. 


\section{NON-COHERENT ML-DETECTION}

As stated in [5] the criterion for non-coherent ML-detection in system given in (1) can be written as

$$
\mathbf{s}_{\mathbf{M L}}=\arg \max _{\mathbf{s} \in \mathcal{S}} \frac{\exp \left(-\operatorname{Tr}\left\{\left[I+k \mathbf{s s}^{*}\right]^{-1} X X^{*}\right\}\right)}{\pi^{m^{2}} \operatorname{det}^{m}\left[I+k \mathbf{s s}^{*}\right]} .
$$

where $k=\rho m$ and $\mathcal{S}=\left\{\frac{e^{\frac{j 2 \pi}{q}}}{\sqrt{m}}, \frac{e^{\frac{j 4 \pi}{q}}}{\sqrt{m}}, \ldots, \frac{1}{\sqrt{m}}\right\}^{m}$. Now, using the matrix inversion lemma and the fact that $\mathbf{s}^{*} \mathbf{s}=1$ we obtain

$$
\begin{array}{r}
\mathbf{s}_{\mathbf{M L}}=\arg \max _{\mathbf{s} \in \mathcal{S}} \frac{\exp \left(-\operatorname{Tr}\left\{\left[I-\frac{1}{k} \mathbf{s s}^{*}\right]^{-1} X X^{*}\right\}\right)}{\pi^{m^{2}}(1+k)^{m}} \\
=\arg \max _{\mathbf{s} \in \mathcal{S}} \operatorname{Tr}\left\{X^{*} \mathbf{s s}^{*} X\right\} .
\end{array}
$$

Therefore, the integer optimization problem one needs to solve can be written as

$$
\max _{\mathbf{s} \in \mathcal{S}} \operatorname{Tr}\left(X X^{*} \mathbf{s s}^{*}\right)
$$

(Since $\mathbf{s}^{*} \mathbf{s}=1$ precisely the same optimization problem is obtained if the criterion of optimization were joint channel estimation and signal detection [12]). Optimization (3) is a very difficult problem. In [6] the case $q=2$ was considered. The sphere decoder algorithm was employed to solve (3) exactly. However, for some parameters of the system, the sphere decoder may be computationally costly. In this paper, we study an alternative algorithm to the sphere decoder which we refer to as out-sphere decoder.

\section{OUT-SPHERE DECODER}

In this section we introduce an exact algorithm for solving (3). The main idea of the algorithm is based on finding all points $\mathrm{s}$ such that $X^{*} \mathrm{~s}$ lies outside a sphere of some adequately chosen radius $d_{s}=\lambda m^{2}$, i.e., on finding all s such that

$$
d_{s}^{2} \leq\left\|X^{*} \mathbf{s}\right\|_{2}^{2}
$$

and then choosing the one that minimizes the objective function. Using the $Q R$-decomposition of $X^{*}=Q R$ ( $Q$ is unitary matrix, $\mathrm{R}$ is upper triangular matrix), we can reformulate (4) as

$$
d^{2} \leq\|R \mathbf{s}\|_{2}^{2} .
$$

Although (5) resembles to the standard sphere decoder used for the minimization problem, it is fundamentally different. To see the main difference let us recall that in the standard sphere decoder applied for minimization of a quadratic form we have

$$
\begin{aligned}
d^{2} & \geq\|R \mathbf{s}\|_{2}^{2}=\left\|R_{m, m} \mathbf{s}_{m}\right\|^{2} \\
& +\left\|R_{1: m-1,1: m-1} \mathbf{s}_{1: m-1}+R_{1: m-1, m} \mathbf{s}_{m}\right\|^{2} \\
\Rightarrow d^{2} & \geq\left\|R_{m, m} \mathbf{s}_{m}\right\|^{2} .
\end{aligned}
$$

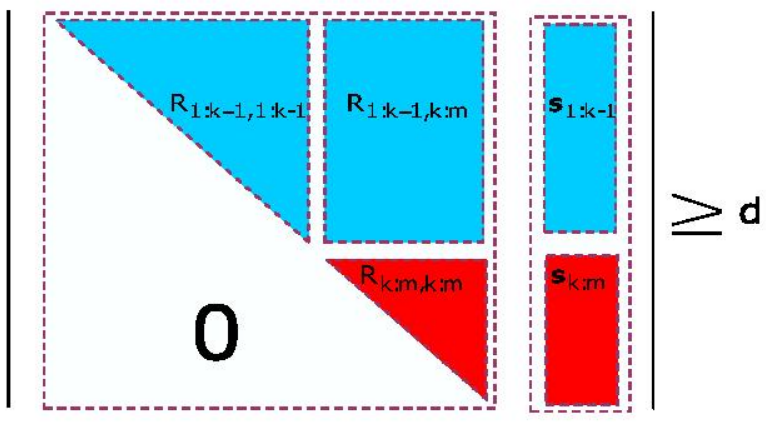

Fig. 2. QR-factorization

However for the maximization problem in (5)

$$
\begin{aligned}
d^{2} & \leq\|R \mathbf{s}\|_{2}^{2}=\left\|R_{m, m} \mathbf{s}_{m}\right\|^{2} \\
& +\left\|R_{1: m-1,1: m-1} \mathbf{s}_{1: m-1}+R_{1: m-1, m} \mathbf{s}_{m}\right\|^{2} \\
\not \Rightarrow d^{2} & \leq\left\|R_{m, m} \mathbf{s}_{m}\right\|^{2} .
\end{aligned}
$$

Therefore in order to find all s such that (5) is satisfied we need a different approach. In what follows we descrive how this problem can be overcome.

Using the upper-triangular property of $R$ (see Figure 2), (5) can be further rewritten as

$$
\begin{aligned}
d^{2} & \leq\left\|R_{k: m, k: m} \mathbf{s}_{k: m}\right\|^{2} \\
& +\left\|R_{1: k-1,1: k-1} \mathbf{s}_{1: k-1}+R_{1: k-1, k: m} \mathbf{s}_{k: m}\right\|^{2},(6)
\end{aligned}
$$

for any $2 \leq k \leq m$, where the subscripts determine the entries the various vectors and matrices run over (e.g. $R_{1: k-1, k: m}$ is a $(k-1) \times(m-k+1)$ matrix and $R_{i, k}, R_{i, k+1}, \ldots, R_{i, m}$ are the components of its $i$-th row). A necessary condition for (5) can therefore be obtained by upper-bounding the second term on the right-hand side (RHS). Let

$$
U B\left(\mathbf{s}_{k: m}\right) \geq \max _{\mathbf{s}_{1: k-1} \in \mathcal{S}^{k-1}}\left\|R_{1: k-1,1: k-1} \mathbf{s}_{1: k-1}+R_{1: k-1, k: m} \mathbf{s}_{k: m}\right\|^{2} .
$$

Then we have a necessary condition for (4)

$$
d^{2} \leq\left\|R_{k: m, k: m} \mathbf{s}_{k: m}\right\|^{2}+U B\left(\mathbf{s}_{k: m}\right) .
$$

The sphere decoder finds all points $\mathrm{s}$ in (4) by proceeding inductively on (7), starting from $k=m$ and proceeding to $k=1$. In other words, for $k=m$ it determines all onedimensional lattice points $\mathbf{s}_{m}$ such that

$$
d^{2} \leq\left|R_{m, m} \mathbf{s}_{m}\right|^{2}+U B\left(\mathbf{s}_{m}\right)
$$

and then, for each such one-dimensional lattice point $\mathbf{s}_{m}$, determines all possible values for $\mathbf{s}_{m-1}$ such that

$$
\begin{aligned}
d^{2} & \leq\left\|R_{m-1: m, m-1: m} \mathbf{s}_{m-1: m}\right\|^{2}+U B\left(\mathbf{s}_{m-1: m}\right) \\
& =\left|R_{m, m} \mathbf{s}_{m}\right|^{2}+\left|R_{m-1, m-1} \mathbf{s}_{m-1}+R_{m-1, m} \mathbf{s}_{m}\right|^{2} \\
& +U B\left(\mathbf{s}_{m-1: m}\right) .
\end{aligned}
$$


This gives all two-dimensional lattice points that satisfy (5); we proceed in a similar fashion until $k=1$. We refer to this algorithm as out-sphere decoder. The out-sphere decoder algorithm thus generates a tree (see Figure 3), where the branches at the $(m-k+1)$ th level of the tree correspond to all $(m-k+1)$-dimensional lattice points satisfying (7). Therefore, at the bottom of the tree (the $m$-th level) all points satisfying (4) are found.

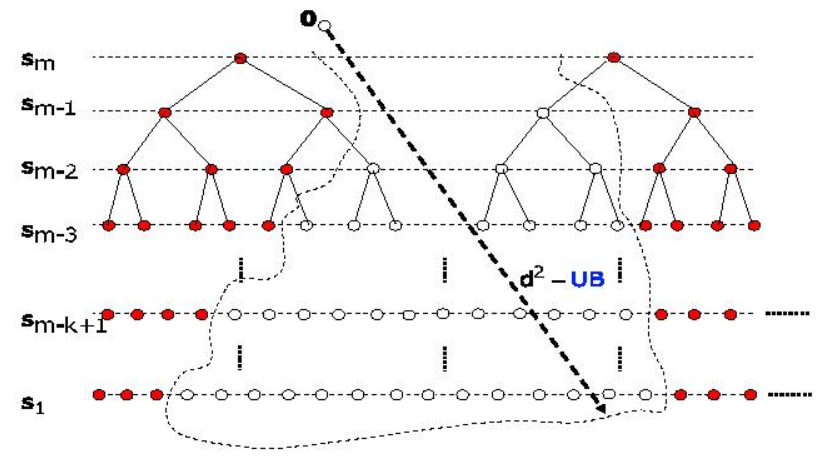

Fig. 3. Tree search

In order to complete the algorithm we need a way of computing $U B\left(\mathbf{s}_{k: m}\right)$. For this we will use well known SDP-relaxation technique (more on SDP-relaxation interested reader can find in [1] and its applications in ML-detection in wireless communications in [7], [11], [9], [8]). Let

$$
\begin{aligned}
U B\left(\mathbf{s}_{k: m}\right) & \geq \max _{\mathbf{s}_{1: k-1} \in \mathcal{S}^{k-1}}\left\|R_{1: k-1,1: k-1} \mathbf{s}_{1: k-1}+R_{1: k-1, k: m} \mathbf{s}_{k: m}\right\|^{2} \\
& =\max _{\mathbf{a}_{k} \in \mathcal{S}^{k}} \mathbf{a}_{k}^{*} Q_{k} \mathbf{a}_{k}=O P T_{k-1}
\end{aligned}
$$

Then SDP dual/relaxation gives

$$
\begin{aligned}
\max _{\mathbf{a}_{k} \in \mathcal{S}^{k}} \mathbf{a}_{k}^{*} Q_{k} \mathbf{a}_{k} \leq U B\left(\mathbf{s}_{k: m}\right)=\min _{\Lambda} & \operatorname{Tr}(\Lambda) \\
\text { subject to } & \Lambda \succeq Q_{k}, \Lambda \text { is diagonal }
\end{aligned}
$$

It can be shown

$$
\alpha O P T_{k-1} \geq U B_{k-1}^{S D P} \geq O P T_{k-1}
$$

It was shown in [2] that if $Q_{k-1} \succeq 0$ is real and $q=2$

$$
\alpha_{r}=\frac{\pi}{2}
$$

It was shown in [3], [4] that if $Q_{k-1} \succeq 0$ is complex

$$
\alpha_{c}=\frac{4 \pi}{\left(q \sin \frac{\pi}{q}\right)^{2}}
$$

Using these results we will now analyze the expected complexity of the out-sphere decoder algorithm.

\subsection{Expected complexity of the out-sphere decoder}

In this section we compute an upper bound the expected complexity of the out-sphere decoder introduced in the previous section. Effectively we will compute the probability that each point in the tree which would correspond to the exhaustive search is actually in the tree of the out-sphere decoder. To make problem tractable we will here make approximative assumption that the matrix $X$ from (3) has i.i.d. real/complex Gaussian entries with zero-mean and unit-variance. In some sense this is an emulation of a very low SNR regime where the matrix $W$ should be dominant in the matrix $X$ and where the complexity of the out-sphere decoder should be the highest. So it is reasonable to believe that in the higher SNR regime that would be of interest in practical consideration the complexity of the out-sphere decoder would be upper-bounded by the value computed based on the assumption that the values of the matrix $X$ from (3) are i.i.d. Gaussian.

\subsubsection{The real case}

In this subsection we will assume $q=2$ and the elements of $\mathbf{h}$ and $W$ are i.i.d real zero-mean unit-variance Gaussian. Now it relatively easily follows that all points from the same level are equally likely to be in the search tree. Let $p_{k}^{j}, 1 \leq$ $j \leq 2^{m-k+1}$ denote the points from the level $m-k+1$ of the search tree $\mathcal{T}$ from Figure 3 . Further let $P_{k}\left(p_{k}^{j} \in \mathcal{T}\right)$ be the probability that $p_{k}^{j}$ is in the search tree $\mathcal{T}$. Clearly the expected complexity of the out-sphere decoder $E C_{\text {osd }}$ can be computed as

$$
E C_{\mathrm{osd}}=\sum_{k=1}^{m} \sum_{j=1}^{2^{m-k+1}} P_{k}\left(p_{k}^{j} \in \mathcal{T}\right) .
$$

Since, $P_{k}\left(p_{k}^{j} \in \mathcal{T}\right)=P_{k}\left(p_{k}^{i} \in \mathcal{T}\right)=P_{k}\left(p_{k} \in \mathcal{T}\right)$, for any $i \neq j$ we have

$$
E C_{\mathrm{osd}}=\sum_{k=1}^{m} 2^{m-k+1} P_{k}\left(p_{k} \in \mathcal{T}\right) .
$$

Now, let us consider in particular the probability that a fixed point from level $k, 0<k \leq m p_{k}$ is in the search tree. Clearly, from (7), (8), (9) we have

$$
\begin{gathered}
P_{k}\left(p_{k} \in \mathcal{T}\right)=\operatorname{Pr}\left(d^{2} \leq\left\|R_{k: m, k: m} \mathbf{s}_{k: m}\right\|^{2}+U B\left(\mathbf{s}_{k: m}\right)\right) \\
\leq \operatorname{Pr}\left(d^{2} \leq\left\|R_{k: m, k: m} \mathbf{s}_{k: m}\right\|^{2}\right. \\
+\alpha_{r} \max _{\mathbf{s}_{1: k-1} \in \mathcal{S}^{k-1}}\left\|R_{1: k-1,1: k-1} \mathbf{s}_{1: k-1}+R_{1: k-1, k: m} \mathbf{s}_{k: m}\right\|^{2} \\
\quad \leq 2^{k-1} \operatorname{Pr}\left(d^{2} \leq\left\|R_{k: m, k: m} \mathbf{s}_{k: m}\right\|^{2}\right. \\
\left.+\alpha_{r}\left\|R_{1: k-1,1: k-1} \mathbf{s}_{1: k-1}+R_{1: k-1, k: m} \mathbf{s}_{k: m}\right\|^{2}\right) .
\end{gathered}
$$

It is not that difficult to note that the two summands on the right hand side of the inequality inside the probability from 
(12) are independent. Hence after applying Chernoff bound we obtain

$$
P_{k}\left(p_{k} \in \mathcal{T}\right) \leq \frac{2^{k-1} E e^{-\mu d^{2}} E e^{\mu\left\|R_{k: m, k: m} \mathbf{s}_{k: m}\right\|^{2}}}{\left(E e^{\mu \alpha_{r}\left\|R_{1: k-1,1: k-1} \mathbf{s}_{1: k-1}+R_{1: k-1, k: m} \mathbf{s}_{k: m}\right\|^{2}}\right)^{-1}}
$$

where $\mu>0$ is Chernoff parameter to be chosen later. Since

$$
\begin{aligned}
\|R \mathbf{s}\|^{2} & =\left\|R_{k: m, k: m} \mathbf{s}_{k: m}\right\|^{2} \\
& +\left\|R_{1: k-1,1: k-1} \mathbf{s}_{1: k-1}+R_{1: k-1, k: m} \mathbf{s}_{k: m}\right\|^{2}
\end{aligned}
$$

we have

$$
\begin{gathered}
E e^{\mu\|R \mathbf{s}\|^{2}}= \\
E e^{\mu\left\|R_{k: m, k: m} \mathbf{s}_{k: m}\right\|^{2}+\mu\left\|R_{1: k-1,1: k-1} \mathbf{s}_{1: k-1}+R_{1: k-1, k: m} \mathbf{s}_{k: m}\right\|^{2}}
\end{gathered}
$$

where $\beta_{c}$ is solution of

$$
2 e^{-\mu m \lambda}\left(\frac{\left(1-2 \mu m \alpha_{r} \beta\right)^{\frac{\beta}{2}}}{(1-2 \mu m \beta)^{\frac{\beta}{2}}\left(1-2 \mu m \alpha_{r}\right)^{\frac{1}{2}}}\right)=2^{\beta_{o}}
$$

and $\mu$ is a parameter to choose so that $\beta_{c}$ is as small as possible. Since optimization over $\mu$ appears to be rather difficult we choose $\mu=\frac{1-\frac{\alpha_{x}}{\lambda}}{2 \alpha_{r} m}$. Finally we have $\beta_{c}$ is the solution of

$$
4 e^{-\left(\frac{\lambda}{\alpha_{r}}-1\right)} \frac{\lambda}{\alpha_{r}}\left(\frac{1-\left(1-\frac{\alpha_{r}}{\lambda}\right) \beta_{c}}{1-\left(1-\frac{\alpha_{r}}{\lambda}\right) \frac{\beta_{o}}{\alpha_{r}}}\right)^{\beta_{o}}=4^{\beta_{o}}
$$

We summarize the results from this subsection in the following theorem.

and by independence of $\left\|R_{1: k-1,1: k-1} \mathrm{~s}_{1: k-1}+R_{1: k-1, k: m} \mathrm{~s}_{k: m}\right\|^{2}$ and $\left\|R_{k: m, k: m} \mathrm{~s}_{k: m}\right\|^{2}$ we further have

$$
\begin{gathered}
\frac{E e^{\mu\left\|R_{1: k-1,1: k-1} \mathbf{s}_{1: k-1}+R_{1: k-1, k: m} \mathbf{s}_{k: m}\right\|^{2}}}{\left(E e^{\mu\left\|R_{k: m, k: m} \mathbf{s}_{k: m}\right\|^{2}}\right)^{-1}}=E e^{\mu\|R \mathbf{s}\|^{2}} \Rightarrow \\
E e^{\mu\left\|R_{1: k-1,1: k-1} \mathbf{s}_{1: k-1}+R_{1: k-1, k: m} \mathbf{s}_{k: m}\right\|^{2}}=\frac{E e^{\mu\|R \mathbf{s}\|^{2}}}{E e^{\mu\left\|R_{k: m, k: m} \mathbf{s}_{k: m}\right\|^{2}}} .
\end{gathered}
$$

Plugging (14) in (13) we obtain

$$
P_{k}\left(p_{k} \in \mathcal{T}\right) \leq \frac{2^{k-1} e^{-\mu d^{2}} E e^{\mu\left\|R_{k: m, k: m} \mathbf{s}_{k: m}\right\|^{2}} E e^{\mu \alpha_{r}\|R \mathbf{s}\|^{2}}}{E e^{\mu \alpha_{r}\left\|R_{k: m, k: m} \mathbf{s}_{k: m}\right\|^{2}}} .
$$

It is straightforward to see that $\|R \mathbf{s}\|^{2}$ is chi-square distributed with $m$ degrees of freedom and $\left\|R_{k: m, k: m} \mathrm{~s}_{k: m}\right\|^{2}$ is chi-square distributed with $m-k+1$ degrees of freedom. Let $\beta=\frac{m-k+1}{m}$. Then we easily obtain

$$
P_{k}\left(p_{k} \in \mathcal{T}\right) \leq 2^{k-1} e^{-\mu d^{2}}\left(\frac{\left(1-2 \mu m \alpha_{r} \beta\right)^{\frac{\beta}{2}}}{(1-2 \mu m \beta)^{\frac{\beta}{2}}\left(1-2 \mu m \alpha_{r}\right)^{\frac{1}{2}}}\right)
$$

Denoting $d^{2}=\lambda m^{2}$ and connecting (11) and (16) we have

$$
E C_{\mathrm{OSd}} \leq \sum_{\beta m=1}^{m} 2^{m} e^{-\mu m^{2} \lambda}\left(\frac{\left(1-2 \mu m \alpha_{r} \beta\right)^{\frac{\beta}{2}}}{(1-2 \mu m \beta)^{\frac{\beta}{2}}\left(1-2 \mu m \alpha_{r}\right)^{\frac{1}{2}}}\right)^{m}
$$

Looking at (17) we note that for certain $\beta$ upper bound on the expected complexity on the righthand side will be larger than $2^{\beta}$. For these $\beta$ s clearly the better choice for upper bound is $2^{\beta}$. However, there will be a critical $\beta_{c}$ such that the upper bound from (17) becomes smaller then the upper bound $2^{\beta}$ obtained from the exhaustive search. It is not that difficult to see that $2^{\beta_{c}}$ will be the highest number of points preserved on average at any level of the tree. Hence we have

$$
E C_{\text {osd }} \leq m 2^{\beta_{\circ} m}
$$

Theorem 1 Consider the SIMO system from (1). Assume that components of $\mathbf{h}$ and $W$ are i.i.d. real Gaussian with zero-mean and unit variance and that $s_{i} \in\left\{-\frac{1}{\sqrt{m}}, \frac{1}{\sqrt{m}}\right\}$. Further assume that the out-sphere decoder is used for solving $M L$-detection problem in a SIMO system described by (1). Its expected complexity $E C_{\text {osd }}$ (averaged over the channel and noise statistics) can be upper bounded in the following way

$$
E C_{o s d} \leq m q^{\beta_{o} m} \text {. }
$$

The constant $\beta_{c}$ can be obtained as solution of

$$
4 e^{-\left(\frac{\lambda}{\alpha_{r}}-1\right)} \frac{\lambda}{\alpha_{r}}\left(\frac{1-\left(1-\frac{\alpha_{r}}{\lambda}\right) \beta_{c}}{1-\left(1-\frac{\alpha_{r}}{\lambda}\right) \frac{\beta_{c}}{\alpha_{r}}}\right)^{\beta_{c}}=4^{\beta_{c}}
$$

where $\lambda=\frac{d_{s}^{2}}{m^{2}}, d_{s}$ is the initial radius, and $\alpha_{r}=\frac{\pi}{2}$ as given in (9).

It is interesting to note that using the replica methods from . statistical physics it was computed in [10]

$$
\lim _{m \rightarrow \infty} \frac{d_{s}^{2}}{m^{2}}=\lambda_{\mathrm{opt}}=\left(\sqrt{\frac{2}{\pi}}+1\right)^{2}
$$

If $\lambda$ in the previous theorem is chosen as $\lambda_{\text {opt }}$ we have the following corollary.

Corollary 1 Assume $\lambda=\left(\sqrt{\frac{2}{\pi}}+1\right)^{2}$ and $m$ is large. Then $\beta_{c}=\frac{2}{3}$ and we obtain an upper bound on the expected complexity of the out-sphere decoder

$$
E C_{\text {OSd }} \leq m 2^{\frac{2 m}{3}} \ll 2^{m} .
$$

The bound obtained in the previous corollary is still exponential. However, the exponent is $2 / 3$ of the exponent in the exhaustive search. 


\subsubsection{The complex case}

Following the similar procedure as above the complex equivalent of the Theorem 1 can be proved. Since the derivation is very similar to the one already presented we only state the final result in the following theorem.

Theorem 2 Consider the SIMO system from (1). Assume that components of $\mathbf{h}$ and $W$ are i.i.d. complex Gaussian with zero-mean and unit variance. Additionally assume that $\mathbf{s} \in\left\{\frac{e^{\frac{j 2 \pi}{q}}}{\sqrt{m}}, \frac{e^{\frac{j 4 \pi}{q}}}{\sqrt{m}}, \ldots, \frac{1}{\sqrt{m}}\right\}^{m}$ and $q$ is an integer power of 2. Further assume that the out-sphere decoder is used for solving ML-detection problem in a SIMO system described by (1). Its expected complexity $E C_{O s d}^{c}$ (averaged over the channel and noise statistics) can be upper bounded in the following way

$$
E C_{\text {osd }}^{c} \leq m q^{\beta_{\circ} m}
$$

The constant $\beta_{c}$ can be obtained as solution of

$$
q e^{-\left(\frac{\lambda}{\alpha_{r}}-1\right)} \frac{\lambda}{\alpha_{r}}\left(\frac{1-\left(1-\frac{\alpha_{r}}{\lambda}\right) \beta_{c}}{1-\left(1-\frac{\alpha_{r}}{\lambda}\right) \frac{\beta_{0}}{\alpha_{r}}}\right)^{\beta_{0}}=q^{\beta_{0}}
$$

where $\lambda=\frac{d_{s}^{2}}{m^{2}}, d_{s}$ is the initial radius, and $\alpha_{c}=\frac{4 \pi}{\left(q \sin \frac{\pi}{q}\right)^{2}}$ as given in (10).

\section{DISCUSSION AND CONCLUSION}

We analyzed a tree search based branch and bound algorithm for solving the non-coherent $\mathrm{ML}$ detection problem in a single-input multiple-output communication systems with q-PSK signalling. An upper bound on the value of the expected complexity of the algorithm is derived.

\section{REFERENCES}

[1] M. Goemans and D. Williamson, "Improved approximation algorithms for maximum cut and satisfiability problems using semidefinite programming," Joumal of the $A C M, 42(6): 1115-11451995$.

[2] Y. Nesterov, "Quality of semidefinite relaxation for nonconvex quadratic optimization," CORE discussion paper, CORE, March 1997.

[3] A.M. So, J. Zhang, Y. Ye, "On Approximating Complex Quadratic Optimization Problems via Semidefinite Programming Relaxations," to appear in Math Programming.

[4] S. Zhang and Y. Huang, "Complex Quadratic Optimization and Semidefinite Programming," SIAM Journal on Optimization, 16(3):871-890, 2006.
[5] B. Hochwald and T. Marzetta, "Unitary space time modulation for multi-antenna modulation in Rayleigh flat fading channel," IEEE Trans. on Inf. Theory, March. 2000.

[6] H. Vikalo, B. Hassibi and P. Stoica, "Joint ML estimation and signal detection," IEEE Trans. on Wireless Communication, July 2006.

[7] W.K. Ma, T.N. Davidson, K.M. Wong, Z.-Q. Luo and P.C. Ching, "Quasi-maximum-likelihood multiuser detection using semi-definite relaxation," IEEE Transactions on Signal Processing, vol. 50, no. 4, pp. 912-922, 2002.

[8] J. Jalden, C. Martin, and B. Ottersten, "Semidefinite Programming for Detection in Linear Systems - Optimality Conditions and Space-Time Decoding," ICASSP, International conference on Acoustics, Signal and Speech Processing, April 2003.

[9] A. Mobasher, M. Taherzadeh, R. Sotirov, and A. Khandani, "A Near Maximum Likelihood Decoding Algorithm for MIMO Systems Based on Semi-Definite Programming," IEEE International Symposium on Information Theory (ISIT'05), Adelaide, Australia, Sept 4-9, 2005 , pp. $1686-1690$

[10] D. Dean and F. Ritort, "Squared interaction matrix Sherrington-Kirkpatrick model for a spin glass," Phys. Rev $B$, vo. 65, 2002.

[11] M. Kisialiou and Z.-Q. Luo, "Performance Analysis of Quasi-Maximum-Likelihood Detector Based on SemiDefinite Programming," Proc. of ICASSP, pp. 433-436, Philadelphia, PA, March 2005.

[12] M.Stojnic, B. Hassibi, and H. Vikalo, "PEP analysis of the SDP-based joint channel estimation and signal detection," ICASSP International Conference on Acoustics, Signal and Speech Processing 2007. 